\title{
The role of particle interactions in a many-body model of Feshbach molecular formation in bosonic systems
}

\author{
Jing $\mathrm{Li}^{1,2,3}$, Di-Fa $\mathrm{Ye}^{2,4}$, Chao $\mathrm{Ma}^{3}$, Li-Bin $\mathrm{Fu}^{2}$, and $\mathrm{Jie}^{2}{ }^{1,2}{ }^{1, *}$ \\ 1. Center for Applied Physics and Technology, Peking University, Beijing 100084, China \\ 2. Institute of Applied Physics and Computational Mathematics, P.O. Box 8009 (28), Beijing 100088, China \\ 3. College of Physics and Information, Beijing Institute of Technology, Beijing 100081, China \\ 4.Graduate School, China Academy of Engineering Physics, Beijing 100088, China
}

\begin{abstract}
In this paper, we investigate the atom-molecule conversion dynamics of a generalized many-body model that includes the atom-atom, atom-molecule, and molecule-molecule interactions, emphasizing the efficiency of the Feshbach molecular formation. We show that the picture of two-body molecular production depicted by the Landau-Zener model is significantly altered: The energy levels are dramatically distorted and the conversion efficiency is suppressed by the particle interactions. According to the rule of constant action and with the help of phase-space analysis, we derive an analytical expression for the conversion efficiency in the adiabatic limit. It shows a ceiling for the conversion efficiency when the interaction strength is larger than a critical value. We further derive a closed equation for the conversion efficiency with the stationary phase approximation. In the sudden limit, the conversion efficiency is twice that predicted by the two-body Landau-Zener formula. Our analytical formula has been confirmed by numerical calculations.
\end{abstract}

PACS numbers: $67.60 . \mathrm{Bc}, 03.75 . \mathrm{Lm}, 82.30 . \mathrm{Nr}$

\section{INTRODUCTION}

The production of ultracold diatomic molecules in bosonic systems is an exciting area of research with important applications ranging from the production of molecular Bose-Einstein condensates (BECs) 1] to the study of chemical reaction dynamics[2]. A widely used production technique involves the association of ultracold atoms into very weakly bound diatomic molecules by applying a time varying magnetic field in the vicinity of a Feshbach resonance 3, 4]. The underlying conversion dynamics are usually described by the Landau-Zener (LZ) model 5]. In this model, the Feshbach molecular production is discussed under a two-body configuration where a single pair of atoms is converted to a molecule at an avoided-crossing between atomic energy level and molecular energy level while the molecular energy is lifted by an applied linearly sweeping magnetic field. Thus, the molecular production efficiency is expected to be an exponential Landau-Zener type 6 , 7].

However, in the above two-body models [6, 7] and their many-body extension [8, 9], interactions between particles such as the atom-atom, atom-molecule, and molecule-molecule interactions, were not taken into account. Recently, Santos et. al. investigated a general many-body two channel model in which the above particle interactions have been taken into account in the Hamiltonian [10]. More interestingly, the inclusion of these particle interactions brings richer dynamics to the Josephson oscillation of the coupled atom-molecule system.

In this work, we extend to study the conversion dy-

*Email: liưjie@iapcm.ac.cn namics of this general many-body model to determine how these interactions affect molecular conversion as the magnetic field is swept across the Feshbach resonance.

In the adiabatic limit, we cast the many-body model into an effective classical Hamiltonian in the meanfield approximation. Such classical analog facilitates us to analyze the conversion dynamics within the frame of classical adiabatic theory [11, 12]. In this framework the conversion dynamics are determined by two scaled parameters $U$ and $\Omega$, where the nonlinear interaction parameter $U$ is proportional to the particle interactions while the coupling parameter $\Omega$ has a close relation to resonance width. We find that the conversion efficiency can be up to $100 \%$ when the scaled nonlinear parameter is smaller than a critical value, i.e., $U / \Omega<\sqrt{2} / 4$, while there is a ceiling of less than $100 \%$ on the conversion efficiency when $U / \Omega>\sqrt{2} / 4$. The ceiling of the conversion efficiency can be calculated analytically according to the principle of constant action. Based on this expression, we can also obtain the scaling laws at the critical point.

In the sudden limit, we derived a closed equation for the conversion efficiency with the stationary phase approximation. We find that the conversion efficiency is twice of that predicted by a standard two-body LandauZener formula when the sweeping rate is very large. This many body effect is consistent with the theoretical analysis based on the renormalized Landau-Zener formula in Ref. 9] that has been used to explain the experimental data[13].

Our paper is organized as follows: In Sec.II we introduce our model. In Sec.III we make a comprehensive analysis of the dynamics of Feshbach molecular formation and show how the particle interactions affect the conversion efficiency. Sec.IV is our conclusion. 


\section{MODEL}

A widely used molecular production technique involves the association of ultracold atom pairs (open channel) into very weakly bound diatomic molecules (close channel) by applying a time varying magnetic field in the vicinity of a Feshbach resonance 3, 4]. Precisely speaking, atoms are converted into molecules at an avoidedcrossing between the atomic energy level and the molecular energy level while the molecular energy is lifted by an applied linearly sweeping magnetic field. The underlying conversion dynamics are properly described by a two channel model. The two-channel model Hamiltonian that includes the atom-atom, atom-molecule, and molecule-molecule interactions, takes following form [10]:

$$
\begin{aligned}
\hat{H}= & \frac{u_{a}}{V} \hat{a}^{\dagger} \hat{a}^{\dagger} \hat{a} \hat{a}+\frac{u_{b}}{V} \hat{b}^{\dagger} \hat{b}^{\dagger} \hat{b} \hat{b}+\frac{u_{a b}}{V} \hat{a}^{\dagger} \hat{a} \hat{b}^{\dagger} \hat{b} \\
& +\epsilon_{a} \hat{a}^{\dagger} \hat{a}+\epsilon_{b} \hat{b}^{\dagger} \hat{b}+\frac{\omega}{\sqrt{V}}\left(\hat{a}^{\dagger} \hat{a}^{\dagger} \hat{b}+\hat{b}^{\dagger} \hat{a} \hat{a}\right),
\end{aligned}
$$

where $\hat{a}^{\dagger}$ is the creation operator for an atomic mode while $\hat{b}^{\dagger}$ creates a molecular mode. $\epsilon_{a}, \epsilon_{b}$ are the chemical potential of the atomic mode and molecular mode, respectively. In experiments, the external magnetic field is linearly swept $B(t)=\dot{B} t$, and crosses the Feshbach resonance at $B_{0}$, thus $2 \epsilon_{a}-\epsilon_{b}=\mu_{c o}\left[B(t)-B_{0}\right]$. Here, $\mu_{c o}$ is the difference between the magnetic moments of a molecule and a pair of separated atoms. $\omega=\sqrt{4 \pi \hbar^{2} a_{b g} \Delta B \mu_{c o} / m}$ denotes the amplitude for the interconversion of atoms and molecules due to the Feshbach resonance, in which $m$ is the mass of a bosonic atom, $a_{b g}$ is the background scattering length, and $\Delta B$ is the width of the resonance. In contrast to the early Hamiltonians [8, 9], we have included the background scattering interactions between atom-atom, moleculemolecule, and atom-molecule, denoted by $u_{a}, u_{b}$, and $u_{a b}$, respectively. $u_{i}=4 \pi \hbar^{2} a_{i} / m_{i}(i=a, b, a b)$ with $a_{i}$ and $m_{i}$ denoting the background scattering length between corresponding particles and their (reduced) mass, respectively. The whole number of the system $N=N_{a}+2 N_{b}$ is a constant and is very large in the present experiments. Here, $N_{a}=\hat{a}^{\dagger} \hat{a}$ and $N_{b}=\hat{b}^{\dagger} \hat{b}$ are the atom number and molecule number, respectively. We introduce the parameter $V$ to denote the quantized volume of the trapped particles, therefore $n=N / V$ is the mean density of the initial bosonic atoms.

Choosing the Fock states as the basis, the Schrödinger equation is written as

$$
i \frac{d}{d t}|\psi\rangle=\hat{H}|\psi\rangle
$$

where $|\psi\rangle=\sum_{i=0}^{N / 2} c_{i}|N-2 i, i\rangle, \quad|N-2 i, i\rangle=$ $\frac{1}{\sqrt{(N-2 i) ! i !}}\left(\hat{a}^{\dagger} \hat{a}^{\dagger}\right)^{N / 2-i}\left(\hat{b}^{\dagger}\right)^{i}|0,0\rangle \quad(i=0, \ldots, N / 2)$ are Fock states, and $c_{i}$ is the probability amplitudes of the corresponding Fock states. The normalization condition is that $\sum_{i}\left|c_{i}\right|^{2}=1$. The Hamitonian matrix elements are $H_{i j}=\langle N-2 i, i|H| N-2 j, j\rangle$. For $i=j$,
$H_{i i}=\frac{(N-2 i)(N-2 i-1)}{V} u_{a}+\frac{i(i-1)}{V} u_{b}+\frac{(N-2 i) i}{V} u_{a b}+(N-$ $2 i) \varepsilon_{a}+i \varepsilon_{b}$; For $i \neq j, H_{i j}=0$ except $H_{i, i+1}=H_{i+1, i}=$ $\sqrt{\frac{(N-2 i)(N-2 i-1)(i+1)}{V}} \omega$.

For the simplest case of $N=2$, the above Schrödinger equation reduces to the following two-level system of standard Landau-Zener type,

$$
i \frac{d}{d t}\left(\begin{array}{l}
c_{0} \\
c_{1}
\end{array}\right)=\left(\begin{array}{ll}
2 \Delta & 2 \Omega \\
2 \Omega & -2 \Delta
\end{array}\right)\left(\begin{array}{l}
c_{0} \\
c_{1}
\end{array}\right)
$$

where $\left|c_{0}\right|^{2}$ and $\left|c_{1}\right|^{2}$ denote the population of atoms and molecules, respectively. The energy bias is $\Delta=$ $\left(2 \epsilon_{a}-\epsilon_{b}+2 n u_{a}\right) / 4$ and the coupling strength is given by $\Omega=\omega \sqrt{n} / 2$. Initially, all particles populate in the lower level of the atomic state, i.e., $c_{0}=1, c_{1}=0$. When the external magnetic field is linearly swept across the Feshbach resonance at $\Delta \simeq 0$, a fraction of atoms will be converted to molecules at the avoided-crossing of energy levels. The conversion efficiency as a function of the sweeping rate (i.e., $\alpha=\dot{\Delta}=\mu_{c o} \dot{B} / 4$ ) and coupling strength, takes the form [5],

$$
\chi=1-\Gamma_{l z}=1-\exp \left(-\frac{2 \pi \Omega^{2}}{\alpha}\right)
$$

It is interesting to notice that in the two-body molecular production picture the particle interactions do not affect the conversion efficiency. This is quite different from the many-body picture as we discuss later.

\section{MANY-BODY EFFECTS $(N \rightarrow \infty)$}

As the total particle number $N$ increases, Eq.(2) is no longer analytically solvable. In addition, the computational demand increases dramatically as $N$ becomes very large. In the mean-field limit where $N \rightarrow \infty$, the quantum fluctuation is negligible. It is appropriate to replace all the quantum operators with c-numbers, thus the Heisenberg equations are casted into the following nonlinear Schrödinger equation,

$$
i \frac{d}{d t}\left(\begin{array}{c}
a \\
b
\end{array}\right)=H\left(\begin{array}{l}
a \\
b
\end{array}\right)
$$

where

$$
H=\left[\begin{array}{cc}
2 U\left(2|b|^{2}-|a|^{2}\right)+\Delta & 4 \Omega a^{*} \\
2 \Omega a & -4 U\left(2|b|^{2}-|a|^{2}\right)-2 \Delta
\end{array}\right],
$$

with

$$
\begin{aligned}
U & =\frac{n}{4}\left(\frac{1}{2} u_{a b}-u_{a}-\frac{1}{4} u_{b}\right) \\
\Delta & =\frac{1}{4}\left(2 \epsilon_{a}-\epsilon_{b}+2 n u_{a}-\frac{1}{2} n u_{b}\right), \\
\Omega & =\frac{\sqrt{n} \omega}{2}
\end{aligned}
$$


and the total population is normalized to a unit, i.e., $|a|^{2}+2|b|^{2}=1$.

We introduce the canonical transformation,

$$
\begin{aligned}
S & =|a|^{2}-2|b|^{2}, \\
\theta & =2 \theta_{a}-\theta_{b},
\end{aligned}
$$

where $\theta_{a}=\arg a$ is the phase of the atomic mode and $\theta_{b}=\arg b$ is the phase of the molecular mode. The quantum system is equivalent to the following classical Hamiltonian,

$$
\mathcal{H}=-2 U S^{2}+2 \Delta S+2 \Omega(1+S) \sqrt{1-S} \cos \theta .
$$

The new canonical variables satisfy

$$
\begin{aligned}
& \frac{d S}{d t}=-\frac{\partial \mathcal{H}}{\partial \theta}=2 \Omega(1+S) \sqrt{1-S} \sin \theta \\
& \frac{d \theta}{d t}=\frac{\partial \mathcal{H}}{\partial S}=-4 U S+2 \Delta+\Omega \frac{1-3 S}{\sqrt{1-S}} \cos \theta
\end{aligned}
$$

Before proceeding, we would like to draw attention to some interesting characteristics of our present Hamiltonian. Compared to the well known nonlinear Hamiltonian that describes the tunneling dynamics of BEC atoms in a double-well potential or between two internal states [14], the nonlinearity (i.e., the Hamiltonian depends on the instantaneous wavefunction as well as its conjugate) of the present Hamiltonian (6) is much more complex. The nonlinearity stems both from the diagonal and off-diagonal terms and is preserved even without taking into account the particle interactions. In addition, the absence of hermicity as well as the lack of $U(1)$ invariance of the Hamiltonian restrict the mean-field motion to a "tear-drop" shaped equal-single-pair-entropy surface 15, 16], rather than the surface of a Bloch sphere. As we will show latter, the interplay of these new features leads to a very different energy level structure and conversion dynamics.

In the following calculations, the coupling constant $\Omega$ is chosen as the energy scale, thus weak nonlinearity and strong nonlinearity refer to $U / \Omega<<1$ and $U / \Omega>>1$, respectively.

\section{A. General properties}

We first show how the nonlinear interactions lead to the deformation of the eigen-energy levels. The eigenstates of the system satisfy that

$$
H\left(\begin{array}{c}
a \\
b
\end{array}\right)=\mu\left(\begin{array}{c}
a \\
2 b
\end{array}\right) .
$$

Solving the above nonlinear equations together with total particle conservation condition $|a|^{2}+2|b|^{2}=1$, we readily obtain the chemical potential $\mu$ and the eigen-state $(a, b)$. The eigen-energies can be derived according to the relationship $\epsilon=\mu / 2+\mu|b|^{2}+\Delta|a|^{2} / 2+4 U|b|^{4}-2 U|a|^{2}|b|^{2}$.
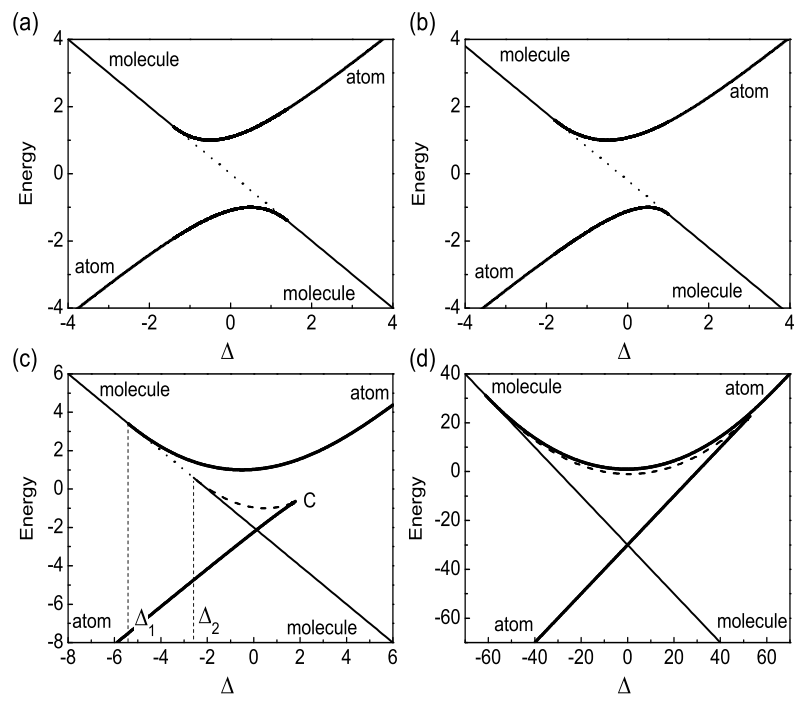

FIG. 1: Adiabatic energy levels for different nonlinear interaction strength: (a) $U=0$, (b) $U=0.2$, (c) $U=2,(\mathrm{~d}) U=30$. In all cases, $\Omega=1$, the solid lines represent stable eigen-states, and the dotted lines between $\Delta_{1}=U-\sqrt{2}$ and $\Delta_{2}=U+\sqrt{2}$ correspond to unstable states. When $U>\sqrt{2} / 4$, a loop structure appears at the lower energy level. The loop expands as $U$ increases.

Their dependence on the parameters is plotted in Fig 1 for the cases of linearity, weak nonlinearity, and strong nonlinearity, respectively. In the linear case $[U=0$, Fig[1(a)], the energy levels are center symmetric. There are only two eigen-states when $|\Delta|$ is large enough, one for atomic mode and the other for molecular mode. When $|\Delta| / \Omega<\sqrt{2}$, there is an additional eigen-state with $S=-1$, represented by the dotted line in Fig 1, This eigen-state is dynamically unstable. With the appearance of nonlinear interaction, the symmetry of the energy levels breaks down. For the weak nonlinear case $U / \Omega<\sqrt{2} / 4$ [see Fig[(b)], the energy level structure is very similar to that of the linear case except for a slight shift. However, when $U / \Omega>\sqrt{2} / 4$, a loop structure appears at the lower energy level. The loop expands as $U$ increases, and the gap between the upper and lower energy level becomes narrower and narrower. Such deformation of energy levels consequently leads to very different conversion dynamics.

Consider the adiabatic evolution of the system starting from the atomic mode at the left side of the lower energy level. When $U$ is small, e.g., in Fig:1(a), the evolution of the system follows the solid line, converting all atoms into molecules. However, when $U / \Omega>\sqrt{2} / 4$ as in Fig.1(c), the system moves steadily from the left side to the critical point $\mathrm{C}$. After that, there is no way to go further except to jump to the upper and lower levels. As that fraction of atoms tunnels to the upper level, they are not converted into molecules. The situation becomes even worse when $U$ is very large: the critical point is much closer to the upper level and far away from the lower one, thus the system will jump to the upper level 


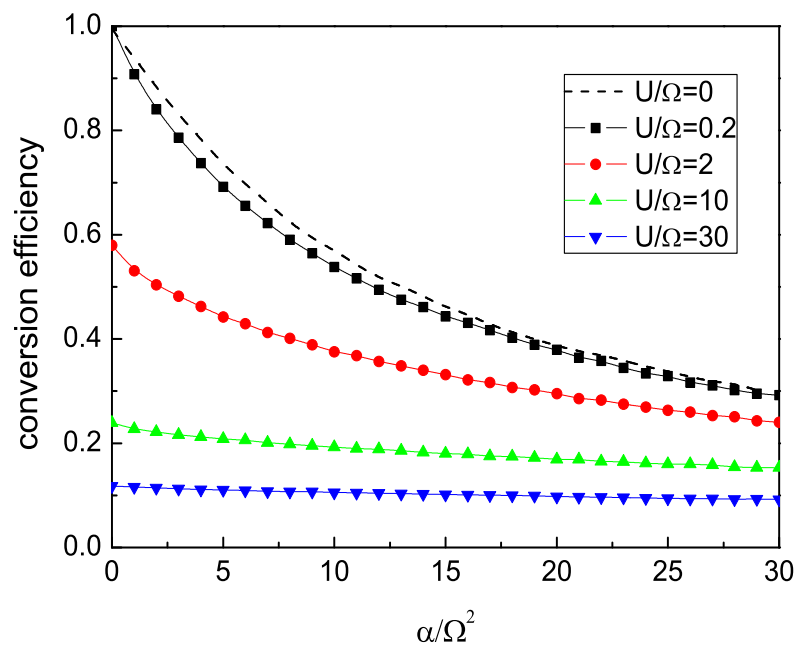

FIG. 2: (Color online) Conversion efficiency as a function of the sweeping rate $\alpha / \Omega^{2}$ for various interaction.

more easily, see Fig 1(d). As a result, almost all atoms can not be converted into molecules.

The above simple analysis is confirmed by our numerical results, which are plotted in Fig 2, In our calculations, the 4-5th Runge-Kutta step-adaptive algorithm is used to solve the nonlinear Schrödinger equation (5). The conversion efficiency as a function of the sweeping rate $\alpha$ is plotted against the nonlinear parameters ranging from weak nonlinearity to strong nonlinearity. Fig 2 shows that (1) the nonlinear interaction always suppresses the conversion from atoms to molecules. For example, in the case of strong nonlinearity $U / \Omega=30$, the conversion efficiency is only about $10 \%$. (2) The conversion efficiency decreases monotonously as the sweeping rate increases. In the following sections, we will further derive analytical formulas for the conversation efficiency in the two limit cases, namely, the adiabatic and sudden limit, corresponding to $\alpha / \Omega^{2}<<1$ and $\alpha / \Omega^{2}>>1$.

\section{B. Adiabatic limit}

In the adiabatic limit where the external field varies slowly compared with the intrinsic motion of the system, the conversion dynamics are entirely determined by the phase-space structure evolution of the classical Hamiltonian (12). The fixed points (i.e., the energy extrema of the classical Hamiltonian) on the phase space correspond to the quantum eigenstates. For example, in Fig 3 (d), the elliptical point (red circle) corresponds to the atomic mode at the left side of the lower energy level in Fig 1(c), and the saddle point (blue circle) corresponds to the unstable eigenstate at the tip of the loop. According to the classical adiabatic theory, when the energy bias $\Delta$ is modulated adiabatically, a closed orbit in the phase-
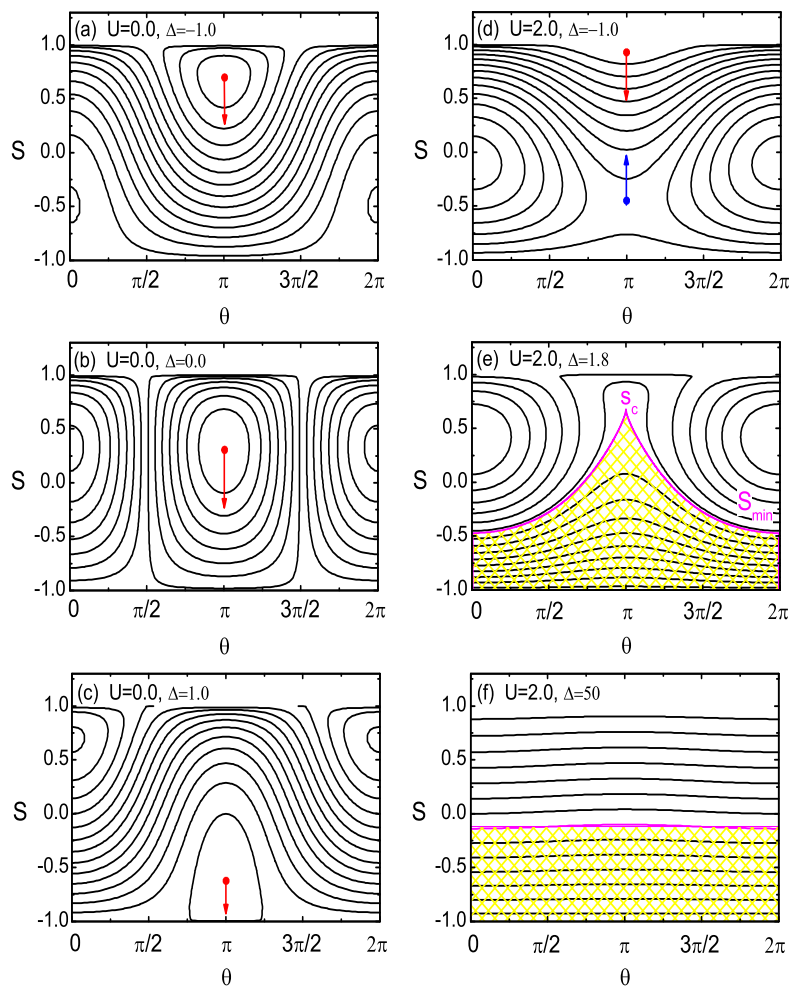

FIG. 3: (Color online) The phase space evolution as $\Delta$ changes adiabatically. The left column and right column represent the linear and nonlinear case, respectively. The arrows indicate the shifting direction of the fixed points as $\Delta$ increases (see text for detail).

space remains closed and the action

$$
I=\frac{1}{2 \pi} \oint S d \theta
$$

stays invariant in time. The action equals the phasespace area enclosed by the closed orbit, and is zero when the orbit shrinks to a fixed point.

For the case of $U / \Omega<\sqrt{2} / 4$, the initial state [atomic mode at the left side of the lower energy level in Fig $1(b)]$ is prepared at an elliptical point on the phase space [red circle in Fig_3(a)]. The elliptical point evolves continuously from the boundary line of $S=1$ to $S=-1$ throughout the entire sweeping of $\Delta$. According to the rule of constant action, it is thus expected that the system follows the elliptical point and finally reaches $S=-1$, implying the entire conversion of atoms into molecules, i.e., the conversion efficiency is $\chi=1$.

However, for the case of $U / \Omega>\sqrt{2} / 4$, the elliptical point [red circle in Fig.3(d)] will collide with a saddle point [blue circle in Fig.3(d)] when $\Delta=\Delta_{c}$ [see Fig.3(e)]. After this collision, the system enters an new obit [magenta line in Fig]3(e)] with $\mathcal{H}=\mathcal{H}_{c}$, and evolves adiabatically for $\Delta>\Delta_{c}$ according to the rule of constant action, which is now nonzero. This obit eventually evolves into a straight line of constant $S$ [magenta line in Figß3(f)]. With these considerations, we can obtain the conversion 


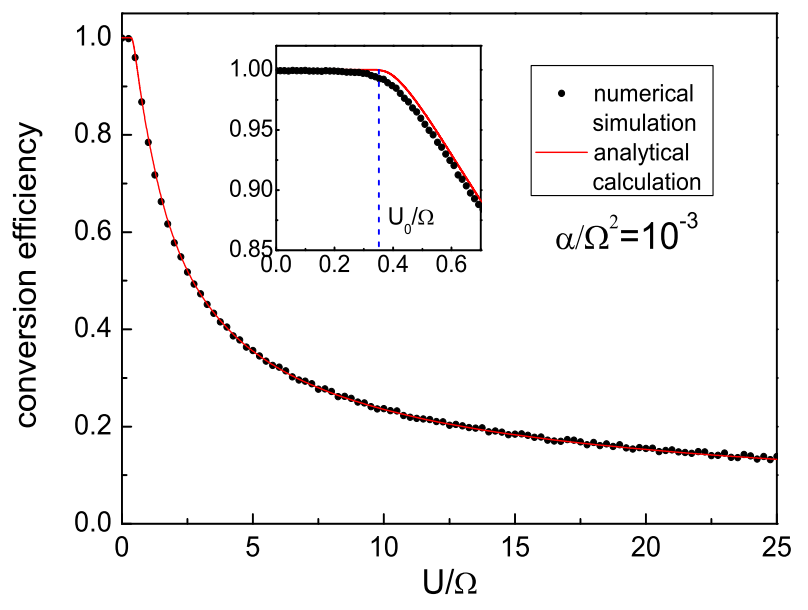

FIG. 4: (Color online) Comparison between analytical calculation (red lines) and numerical simulation (full circles) in the adiabatic limit, excellent agreement is clearly seen. $U_{0} / \Omega=\sqrt{2} / 4$.

efficiency in the adiabatic limit,

$$
\chi=1-\frac{1}{2} I_{c} .
$$

To work out the explicit expression of $\chi$, we first need to determine the critical point $\mathrm{C}$. For this purpose, we notice that point $\mathrm{C}$ (with $\theta=\pi$ ) is a double root of Eq.(13) (14), thus

$$
\left.\frac{\partial \dot{\theta}}{\partial S}\right|_{S_{c}}=-4 U+\Omega \frac{5-3 S_{c}}{2\left(1-S_{c}\right)^{3 / 2}}=0 .
$$

The critical energy bias $\Delta_{c}$ and obit energy $\mathcal{H}_{c}$ can be obtained through Eq.(14) and Eq.(12), respectively

$$
\begin{aligned}
& \Delta_{c}=2 U S_{c}+\Omega \frac{1-3 S_{c}}{2 \sqrt{1-S_{c}}}, \\
& \mathcal{H}_{c}=-2 U{S_{c}}^{2}+2 \Delta_{c} S_{c}-2 \Omega\left(1+S_{c}\right) \sqrt{1-S_{c}} .
\end{aligned}
$$

Once these critical values are given, the whole orbit [magenta line in Fig[3(e)] passing through the critical point can be determined by

$$
\cos \theta=\frac{\mathcal{H}_{c}+2 U S^{2}-2 \Delta_{c} S}{2 \Omega(1+S) \sqrt{1-S}} .
$$

We calculate the action by changing the parameter of the integration,

$$
\begin{aligned}
I_{c}= & \frac{1}{2 \pi} \oint \theta d S \\
= & \frac{1}{\pi} \int_{S_{\text {min }}}^{S_{c}}\left[\pi-\arccos \left(\frac{\mathcal{H}_{c}+2 U S^{2}-2 \Delta_{c} S}{2 \Omega(1+S) \sqrt{1-S}}\right)\right] d S \\
& +1+S_{\text {min }} .
\end{aligned}
$$

Here, $S_{\min }$ can be determined by Eq.(21) with $\theta=0$, i.e.,

$$
1=\frac{\mathcal{H}_{c}+2 U S_{\min }^{2}-2 \Delta_{c} S_{\min }}{2 \Omega\left(1+S_{\min }\right) \sqrt{1-S_{\min }}}
$$

The results based on the above analytical calculation are compared with those obtained from numerical simulation in Fig 4 and excellent agreement is obtained. When $U / \Omega<\sqrt{2} / 4$ the conversion efficiency is always a constant, while when $U / \Omega>\sqrt{2} / 4$ the conversion efficiency decreases suddenly. This implies a phase transition at the critical point of $U_{0} / \Omega=\sqrt{2} / 4$. To confirm the occurrence of phase transition and determine its order, we first need to obtain the scaling law around the critical point. For this purpose, we introduce a small variable $\delta=U / \Omega-\sqrt{2} / 4$, and calculate the critical values with perturbation theory,

$$
\begin{aligned}
S_{c} & =-1+\frac{16 \sqrt{2}}{3} \delta-\frac{256}{9} \delta^{2}+\frac{5888 \sqrt{2}}{81} \delta^{3}, \\
\Delta_{c} / \Omega & =\frac{\sqrt{2}}{2}-2 \delta+\frac{16 \sqrt{2}}{3} \delta^{2}-\frac{512}{27} \delta^{3}, \\
\mathcal{H}_{c} / \Omega & =-\frac{3 \sqrt{2}}{2}+2 \delta-\frac{32 \sqrt{2}}{3} \delta^{2}+\frac{2048}{27} \delta^{3} .
\end{aligned}
$$

The critical orbit is determined by (let $S=-1+x$ ),

$$
\cos \theta=-1+\frac{x\left(x-\frac{16 \sqrt{2}}{3} \delta\right) \delta+\frac{512}{27} \delta^{3}}{\sqrt{2} x} .
$$

Here $x_{\max } \sim \frac{16 \sqrt{2}}{3} \delta, x_{\min } \sim \frac{128 \sqrt{2}}{27} \delta^{3}$. The integration of Eq.(22) results in a power law,

$$
\chi \sim 1-2.4 \delta^{2}=1-2.4\left(\frac{U}{\Omega}-\frac{\sqrt{2}}{4}\right)^{2}, \frac{U}{\Omega} \rightarrow \frac{\sqrt{2}}{4} .
$$

Clearly, both $\chi$ and its first-order derivative are continuous at the critical point. However, its second-order derivative turns to be discontinuous, indicating the appearance of a second order phase transition at the critical point.

The power law at the asymptotic regime of $U / \Omega \rightarrow \infty$ can also be obtained with the above treatment. In this case, we introduce a new small variable $\lambda=(U / \Omega)^{-1}$ and use this small variable to expand the critical values, $S_{c}=1-\frac{1}{4}(2 \lambda)^{2 / 3}, S_{\min }=1-\frac{9}{4}(2 \lambda)^{2 / 3}, \Delta_{c} / \Omega=$ $2 \lambda^{-1}-\frac{3 \sqrt[3]{4}}{2} \lambda^{-1 / 3}+\frac{3 \sqrt[3]{2}}{4} \lambda^{1 / 3}, \mathcal{H}_{c} / \Omega=2 \lambda^{-1}-3 \sqrt[3]{4} \lambda^{-1 / 3}+$ $\frac{3 \sqrt[3]{2}}{4} \lambda^{1 / 3}$. After integrating Eq.(22), we finally come to a power law of the conversion efficiency,

$$
\chi \sim 1.2 \lambda^{2 / 3}=1.2\left(\frac{U}{\Omega}\right)^{-2 / 3}, \frac{U}{\Omega}>>1 .
$$

We would like to mention that, in the nonlinear Landau-Zener model describing the tunneling dynamics of BEC atoms in a double-well potential or between two internal states, the power laws at the critical and asymptotic regime are very different [14]. At the critical regime, both the prefactor and exponent of the power law is different from that in Eq.(28). While at the asymptotic regime, only the prefactor is different compared with Eq.(29). 


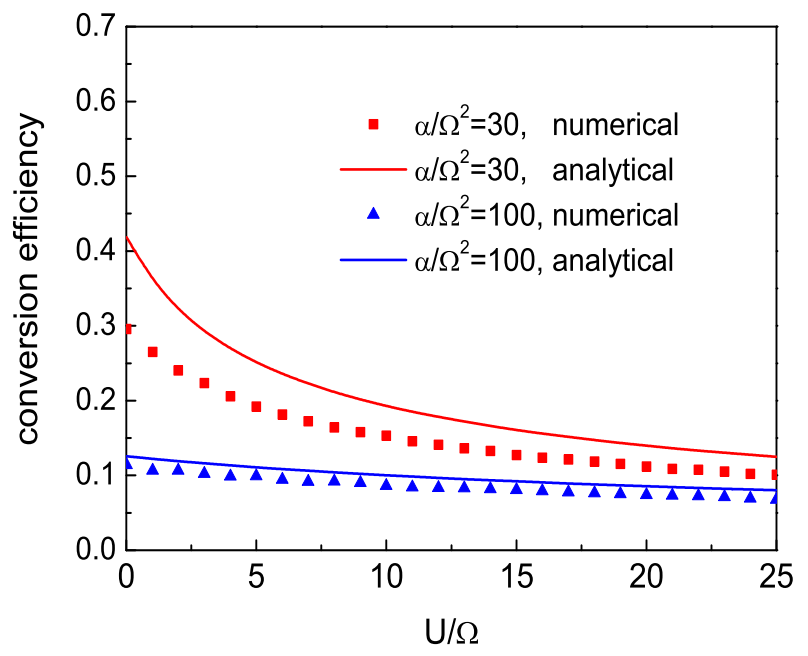

FIG. 5: (Color online) Comparison between analytical calculation (solid lines) and numerical simulation (scatters) in the sudden limit. Good agreement is obtained, especially for the rapidly sweeping case $\alpha / \Omega^{2}=100$.

\section{Sudden limit}

The sudden limit corresponds to nonadiabatic conversion. The conversion efficiency is not strongly related to the structure of the energy levels. In this limit, we can derive the analytical expression of the conversion efficiency using the stationary phase approximation (SPA). Because of the large sweeping rate $\alpha$, a quantum state would stay on the initial level most of the time. Thus the amplitude $b$ in the Schrödinger equation (5) remains small and $|a| \sim 1$ all the time. A perturbation treatment of the problem becomes adequate.

We begin with the variable transformation,

$$
\begin{aligned}
& a=a^{\prime} \exp \left\{-i \int_{0}^{t}\left[\Delta+2 U\left(2|b|^{2}-|a|^{2}\right)\right] d t\right\}, \\
& b=b^{\prime} \exp \left\{i \int_{0}^{t}\left[2 \Delta+4 U\left(2|b|^{2}-|a|^{2}\right)\right] d t\right\} .
\end{aligned}
$$

As a result, the diagonal terms in the Hamiltonian are transformed away, and the evolution equation of $b^{\prime}$ becomes:

$\frac{d b^{\prime}}{d t}=-2 i \Omega\left(a^{\prime}\right)^{2} \exp \left\{-i \int_{0}^{t}\left[4 \Delta+8 U\left(2|b|^{2}-|a|^{2}\right)\right] d t\right\}$,

here $a^{\prime} \sim 1$ all the time and $|a|^{2}+2|b|^{2}=1$, thus

$b^{\prime}=-2 i \Omega \int_{-\infty}^{t} d t \exp \left\{-i \int_{0}^{t}\left[4 \Delta+8 U\left(4|b|^{2}-1\right)\right] d t\right\}$.

We need to calculate the above integral selfconsistently. Due to the large $\alpha$, the nonlinear term in the exponent generally gives a rapid phase oscillation, which makes the integral small. The dominant contribution comes from the stationary point $t_{0}$ of the phase around which we have

$$
4 \Delta+8 U\left(4|b|^{2}-1\right)=\bar{\alpha}\left(t-t_{0}\right)
$$

with

$$
\bar{\alpha}=4 \alpha+32 U\left[\frac{d|b|^{2}}{d t}\right]_{t_{0}} .
$$

Since $|b|^{2}=\left|b^{\prime}\right|^{2}$, then we have

$$
|b|^{2}=(2 \Omega)^{2}\left|\int_{-\infty}^{t} d t \exp \left[\frac{i}{2} \bar{\alpha}\left(t-t_{0}\right)^{2}\right]\right|^{2} .
$$

This expression can be differentiated and evaluated at time $t_{0}$. A standard Fresnel integral with the result $\left[d|b|^{2} / d t\right]_{t_{0}}=(2 \Omega)^{2} \sqrt{\pi / \bar{\alpha}}$ is obtained. Combining this with relation (35), we come to a closed equation for $\bar{\alpha}$,

$$
\bar{\alpha}=4 \alpha+32 U(2 \Omega)^{2} \sqrt{\frac{\pi}{\bar{\alpha}}} .
$$

The conversion efficiency

$$
\chi=2|b|_{+\infty}^{2}=\frac{16 \pi \Omega^{2}}{\bar{\alpha}} .
$$

Substituting it into Eq.(37), we obtain the analytical expression for the conversation efficiency,

$$
\frac{1}{\chi}=\frac{\alpha}{4 \pi \Omega^{2}}+\frac{2 U}{\pi \Omega} \sqrt{\chi} .
$$

In Fig 5, we have calculated the conversion efficiencies using Eq.(39) and compared them with the numerical results obtained by directly integrating the Schrödinger equation (5), where a good agreement is shown, especially for the rapidly sweeping case, e.g., $\alpha / \Omega^{2}=100$. In this case, the second term on the right hand side of Eq.(39) can be neglected, thus we get $\chi=4 \pi \Omega^{2} / \alpha$. This result from our many-body mean-field theory is twice that obtained from the standard two-body Landau-Zener formula, i.e., the first order expansion of Eq.(4). This many body effect is consistent with the theoretical analysis based on a renormalized Landau-Zener formula in Ref.[9].

\section{CONCLUSION AND DISCUSSION}

In conclusion, we have both numerically and analytically investigated a generalized many-body model that includes the atom-atom, atom-molecule, and moleculemolecule background scattering interactions, emphasizing the dynamics of Feshbach molecular formation. Compared to the simple two-body molecular production picture depicted by the Landau-Zener model, the conversion dynamics become more complicated because of the 
interplay of the many-body effect and the nonlinear interaction effect.

In the adiabatic limit, the nonlinear particle interactions dominate while in the sudden limit the many-body effect becomes significant. In the adiabatic limit, the energy level structure is dramatically distorted due to the particle interactions. Especially when the nonlinear parameter goes beyond a critical value, a loop appears at the tip of the lower energy level. This loop structure consequently leads to the break down of adiabaticicy, and correspondingly a ceiling of less than $100 \%$ on the conversion efficiency. The above striking phenomenon emerges when the effective interaction parameter $U / \Omega$ is large. Because the above parameter is proportional to the square root of the density and inversely proportional to the square root of the resonance width, the above phenomena are expected to be observable in experiments performed with atom clouds of relative high density and narrow Feshbach resonance width.
In the sudden limit, we derived a closed equation for the conversion efficiency with the stationary phase approximation. We found that the conversion efficiency is twice that predicted by the standard two-body LandauZener formula when the sweeping rate is very large. This many-body effect is consistent with the theoretical analysis based on a renormalized Landau-Zener formula in Ref.[9], that has been exploited to explain the experimental data of JILA [13] and agrees well.

\section{ACKNOWLEDGMENTS}

This work is supported by National Natural Science Foundation of China (No.10725521,10604009), the National Fundamental Research Programme of China under Grant No. 2006CB921400, 2007CB814800.
[1] S. Inouye, M. R. Andrews, J. Stenger, H. -J. Miesner, D. M. Stamper-Kum, and W. Ketterle, Nature (London) 392, 151 (1998).

[2] D. J. Heinzen and Roahn Wynar, P. D. Drummond and K. V. Kheruntsyan, Phys. Rev. Lett. 84, 5029 (2000).

[3] E. Timmermans, P. Tommasini, M. Hussein, and A. Kerman, Phys. Rep. 315, 199 (1999).

[4] Thorsten Köhler, Krzysztof Góral, and Paul S. Julienne Rev. Mod. Phys. 78, 1311 (2006).

[5] L. D. Landau, Phys. Z. Sowjetunion 2, 46 (1932); G. Zener, Proc. R. Soc. London, Ser. A 137, 696 (1932).

[6] F. H. Mies, E. Tiesinga, and P. S. Julienne, Phys. Rev. A 61, 022721 (2000).

[7] Krzysztof Goral, Thorsten Koehler, Simon A. Gardiner, Eite Tiesinga, Paul S. Julienne, J. Phys. B 37, 3457 (2004).

[8] A. Vardi, V. A. Yurovsky, and J. R. Anglin, Phys. Rev. A 64, 063611 (2001).

[9] Jie Liu, Bin Liu and Li-Bin Fu, arXiv:0712.4191 to ap- pear in Phys. Rev. A.

[10] G. Santos, A. Tonel, A. Foerster, and J. Links, Phys. Rev. A 73, 023609 (2006).

[11] L.D. Landau and E.M. Lifshitz, Mechanics (Pergamon, Oxford, 1977).

[12] Jie Liu, Biao Wu, and Qian Niu, Phys. Rev. Lett. 90, 170404 (2003).

[13] E. Hodby, S. T. Thompson, C. A. Regal, M. Greiner, A. C. Wilson, D. S. Jin, E. A. Cornell, and C. E. Wieman, Phys. Rev. Lett. 94, 120402 (2005).

[14] Jie Liu, Li-Bin Fu, Bi-Yiao Ou, Shi-Gang Chen, and Qian Niu, arXiv:quant-ph/0105140v1; Jie Liu, Libin Fu, BiYiao Ou, Shi-Gang Chen, Dae-Il Choi, Biao Wu and Qian Niu, Phys. Rev. A 66, 023404 (2002).

[15] I. Tikhonenkov, E. Pazy, Y. B. Band, M. Fleischhauer, and A. Vardi, Phys. Rev. A 73, 043605 (2006).

[16] A. P. Itin and S. Watanabe, Phys. Rev. E 76, 026218 (2007). 